\title{
Adverse Effects of Checkpoint Inhibitor Immunotherapy in Medical Oncology
}

\author{
Michael Brister, MD and Colin Thomas, MD
}

\begin{abstract}
INTRODUCTION
The development of checkpoint inhibitor immunotherapy marks a significant innovation in the field of medical oncology over the past decade. Checkpoint inhibitors are antibody drugs that have demonstrated efficacy in treating a wide range of malignancies including advanced melanoma, non-small cell lung cancer, and Hodgkin lymphoma. These drugs antagonize cell-associated molecules responsible for immunologic down-regulation including programmed cell death receptor 1 (PD-1), programmed cell death ligand 1 (PD-L1) and cytotoxic T-lymphocyte-associated antigen 4 (CTLA-4). Curtailing the function of these intrinsic immunologic down-regulators enhances the body's own antitumor immune mechanisms. Despite the promise of these new therapies, a variety of immunerelated adverse events (irAEs) have been described. ${ }^{1,2}$ Utilization of immunologic checkpoint blockade is increasing in medical oncology, and the importance of understanding these new toxicities is evident. Here we discuss some of the most important adverse effects of checkpoint inhibitors and review the basic principles of managing these conditions. There is a lack of strong evidence guiding the optimal management of irAEs, but treatment algorithms exist based on clinical experience.
\end{abstract}

\section{GASTROINTESTINAL TOXICITY}

The most common gastrointestinal irAEs are diarrhea and colitis. Symptoms generally develop about 6-8 weeks after treatment initiation. ${ }^{3}$ Whereas diarrhea is based on an increase in stool frequency or ostomy output compared to baseline, indicators of colitis include stools with blood or mucus, or the presence of abdominal pain and cramping. Enteritis involves symptoms similar to colitis, but anatomically the small bowel is affected. Esophagitis and gastritis have also been reported as irAEs, but are much less common. Gastrointestinal irAEs are more frequent in patients treated with CTLA-4 targeting therapy such as ipilimumab, compared with PD-L1 or PD-1 inhibitors such as Nivolumab. ${ }^{4.5}$ In a study of patients with melanoma receiving ipilimumab monotherapy, 28\% developed diarrhea and $8 \%$ developed colitis. Interestingly, 5\% of patients developed severe (grade 3/4) colitis. 4.6 Gastrointestinal irAEs frequently reach grade 3/4 in severity, thus early recognition and appropriate management is important to reduce the risk of life-threatening complications such as bowel perforation.
Patients that develop diarrhea or abdominal pain while on checkpoint inhibitors should first be evaluated for infectious diarrhea including $C$. difficile. If infectious diarrhea can be excluded and symptoms persist or worsen, an adverse reaction to immunotherapy becomes more likely. However, it is difficult to make this distinction definitively and treatment is often empiric. The Common Terminology Criteria for Adverse Events (CTCAE) defines grade 1 diarrhea as an increase of fewer than four stools per day above baseline, and patients with grade 1 colitis are usually asymptomatic. These patients are treated symptomatically with loperamide or diphenoxylate and atropine, and immunotherapy may be resumed as scheduled with close monitoring and patient education. Grade 2 diarrhea is defined as four to six stools per day above baseline, and grade 2 colitis is defined by the presence of abdominal pain or bloody stools. For these patients, checkpoint inhibitor therapy should be stopped until symptoms improve to grade 1 with symptomatic management. However, if symptoms persist for more than 5 days, systemic corticosteroids should be begun at a dose of $0.5-1.0 \mathrm{mg} / \mathrm{kg} /$ day of intravenous (IV) methylprednisolone or the oral equivalent. In this case, colonoscopy or computed tomography (CT) evaluation is helpful to confirm the diagnosis. If symptoms worsen or do not improve within 3 days of steroid treatment, the diarrhea or colitis should be managed as grade 3/4 with 1.0-2.0 mg/kg/day of IV methylprednisolone, and the immunotherapy should be permanently discontinued. Steroids may only be tapered after symptoms improve to grade 1, and the taper should be performed slowly over at least 1 month. If symptoms do not improve to grade 1 and persist for more than 3 days despite higher steroid dosing, it is recommended to add infliximab to the treatment regimen, similar to the management of active inflammatory bowel disease (IBD). ${ }^{7}$ Grade 3/4 diarrhea is defined as seven or more stools per day above baseline, and grade $3 / 4$ colitis includes severe abdominal pain, sometimes with peritoneal signs. If intestinal perforation has occurred, infliximab use is contraindicated.

Patients with hepatotoxicity from checkpoint inhibitor therapy are usually asymptomatic and patients are diagnosed based on increased levels of aspartate aminotransferase (AST), alanine aminotransferase (ALT), and/or total bilirubin noted 8-12 weeks after immunotherapy initiation. Occasionally, mild symptoms are present including fatigue or fever, but death from fulminant hepatitis has also been reported. ${ }^{8}$ Grade 2 hepatotoxicity is based on measurement of AST or ALT 
2.5-5 times the upper limit of normal, or total bilirubin 1.5-3 times the upper limit of normal. Grade 3 or grade 4 hepatotoxicity is defined as AST or ALT greater than 5 times the upper limit of normal or total bilirubin greater than 3 times the upper limit of normal. In the CheckMate 067 phase III trial, both nivolumab monotherapy and ipilimumab monotherapy were associated with an $8 \%$ incidence of hepatotoxicity based on any grade of transaminase elevation, and grade 3/4 transaminase elevations occurred in $2 \%$ of both patient groups. ${ }^{9}$ Patients receiving combination nivolumab plus ipilimumab had a much higher rate of transaminitis, with $33 \%$ of patients developing hepatic injury of any grade.

Management should include exclusion of other causes of hepatitis, including medication-induced hepatic injury or viral hepatitis. As with other irAEs, immunotherapy should be delayed if grade 2 toxicity is present. With grade 3 or 4 hepatotoxicity, immunotherapy should be discontinued indefinitely and steroids should be started at a dose of 1.0-2.0 mg/kg/day of IV methylprednisolone or oral equivalent. Mycophenolate mofetil may be added if there is no improvement within 3 days. Unlike treatment of severe immunotherapy-related diarrhea or colitis, grade 3/4 hepatotoxicity should not be treated with infliximab, as this drug has been independently implicated as a cause of medication-induced hepatotoxicity.

\section{PNEUMONITIS}

Pneumonitis is a potentially fatal complication of checkpoint inhibitor immunotherapy. This is a particularly important consideration in patients with compromised pulmonary reserve including those who have had radiation therapy, lung resection, or patients with a history of exposure to chemotherapies associated with pneumonitis such as bleomycin, docetaxel, or gemcitabine. In an early phase study of nivolumab monotherapy, deaths due to pneumonitis were reported in 2 patients with non-small cell lung cancer and 1 patient with colorectal cancer, in total representing $1 \%$ of patients in this study. ${ }^{10}$ Pneumonitis caused by checkpoint inhibitors can be difficult to diagnose and the clinical manifestations are diverse. In one study, pneumonitis presented up to 19 months after initiation of checkpoint inhibitor therapy, with a median time to onset of about 3 months. However, one-third of patients were asymptomatic at the time of diagnosis and were identified based on radiologic findings alone, suggesting an even later median time to onset clinically. ${ }^{11}$ When symptoms were present, patients presented with dyspnea, cough, fever, or chest pain.

Before diagnosing immunotherapy-related pneumonitis, more common etiologies of pulmonary symptoms must be excluded. Caution should be taken to rule out infection, often in association with an infectious disease consultation. Previously undiagnosed malignancy infiltrating the lungs must also be considered, and pulmonary consultation is advised when there is concern for grade 2 pneumonitis (mild to moderate new symptoms). CT imaging is helpful to guide diagnosis, and bronchoscopy or lung biopsy may also be warranted, but there are no radiographic or pathologic findings specific to pneumonitis caused by checkpoint inhibitor exposure.

Grade 1 toxicity involves radiographic changes only and requires repeat imaging every 3 weeks to assess if immunotherapy should be delayed. Grade 2 toxicity should be managed with $1.0 \mathrm{mg} / \mathrm{kg} /$ day of methylprednisolone or the oral equivalent, and doses of 2.0-4.0 $\mathrm{mg} / \mathrm{kg} /$ day are recommended for grade 3 or higher toxicity. Prophylactic antibiotics for Pneumocystis pneumonia (PCP) should be added if the patient will require the equivalent of $20 \mathrm{mg}$ of prednisone daily for greater than 4 weeks. If patients do not improve after 48 hours of steroid therapy, additional immunosuppression with infliximab, cyclophosphamide, intravenous immunoglobulin, or mycophenolate mofetil should also be considered. In a group of 43 patients receiving PD-1/ PD-L1 inhibitor therapy who were diagnosed with immunotherapy-related pneumonitis, 86\% improved with either corticosteroid administration or discontinuation of immunotherapy. Of the remaining 6 patients, 5 died from either infection or cancer progression.11 In a comparison of PD-1 inhibitors to PD-L1 inhibitors for the treatment of non-small cell lung cancer, PD-1 inhibitors were found to be more than twice as likely as PD-L1 inhibitors to cause pneumonitis (3.6\% versus $1.3 \%$ ). ${ }^{12}$

\section{ENDOCRINOPATHIES}

The endocrine system relies on finely tuned quantities of hormones controlling target organs, and the body is especially sensitive to altered levels of endocrine signaling. Unlike other organ systems adversely affected by immune checkpoint blockade, endocrine organ dysfunction from checkpoint inhibitors does not readily reverse with corticosteroids. ${ }^{13}$ Life-long hormone supplementation is required in many patients with endocrine irAEs. In a phase 3 trial of patients receiving ipilimumab and nivolumab, more than $50 \%$ of patients who developed any grade of endocrine toxicity required long-term hormone supplementation. Even so, substantial improvement in symptoms was observed. ${ }^{9}$

Endocrinopathies can present a diagnostic challenge because patients develop non-specific complaints including fatigue, weakness, nausea, and abdominal pain. The anterior pituitary is critical for thyroid function, gonadal function, and adrenal activity. The most common endocrine irAEs are hypothyroidism, hyperthyroidism, and hypophysitis. In a study of patients receiving 
ipilimumab for melanoma, 8\% developed hypophysitis and $6 \%$ developed thyroiditis. ${ }^{14}$ There is variability in the time to onset of endocrine-related adverse effects, with a trend towards later presentations compared with irAEs affecting other organ systems. In patients receiving ipilimumab, the median time to onset of hypophysitis was observed to be between 4-8 weeks after treatment initiation, but patients have been diagnosed with immunotherapy-related thyroiditis up to 3 years after treatment initiation, including long after checkpoint inhibitor therapy has been discontinued. ${ }^{14,15}$

Patients suspected to have endocrine irAEs should have laboratory testing of thyroid-stimulating hormone (TSH), triiodothyronine (T3), thyroxine (T4), adrenocorticotropic hormone (ACTH), follicle-stimulating hormone (FSH), luteinizing hormone (LH), and cortisol. These tests should be repeated at regular intervals in patients diagnosed with endocrine irAEs. If there is concern for hypophysitis, the patient should undergo visual field testing and pituitary MRI should be considered to assist in confirming the diagnosis. Patients with hypophysitis who become symptomatic should be treated with $1.0-2.0 \mathrm{mg} / \mathrm{kg} / \mathrm{day}$ of methylprednisolone or the oral equivalent. Immunotherapy treatments should be delayed and appropriate hormone replacement therapy should be started. If the complication is grade 4 (life-threatening), checkpoint inhibitor treatments must be permanently discontinued. Prophylactic antibiotics for PCP should be considered, and if symptoms improve, steroids may be tapered over a minimum of 1 month, but usually longer.

Thyroiditis secondary to checkpoint inhibitors often presents similarly to Hashimoto's thyroiditis with a period of clinical hyperthyroidism preceding a progressive transition towards hypothyroidism over several weeks. In cases of asymptomatic TSH elevation, immunotherapy may be continued; however, patients with a TSH that is less than half the lower limit of normal, or greater than 2 times the upper limit of normal, should have free T4 testing with each cycle of immunotherapy. If hypothyroidism is present, the patient should be started on levothyroxine, but in contrast to the treatment of most irAEs, it is unclear if steroids are helpful in the long-term management of symptomatic thyroiditis.

Less common endocrine irAEs include primary adrenal insufficiency and type 1 diabetes mellitus, which have each been observed in less than $1 \%$ of patients. 16,17 The development of adrenal crisis is a dangerous complication that must be differentiated from sepsis and immediately treated with intravenous corticosteroids. Immunotherapy-related endocrinopathies are variable in presentation, often permanent, and require careful consideration in patients exposed to checkpoint inhibitors presenting with vague systemic complaints.

\section{CONCLUSION}

Immunotherapy is becoming increasingly prevalent in medical oncology. Studies have indicated checkpoint inhibitors are better tolerated and associated with fewer adverse effects overall in comparison with standard chemotherapy, ${ }_{18}$ but the toxicities associated with immunotherapy are often difficult to recognize and may affect several organ systems. The most important irAEs are diarrhea, colitis, hepatitis, pneumonitis, and endocrinopathies. The skin, however, is the most commonly involved organ, and the most frequently observed cutaneous toxicity is a mild pruritic maculopapular rash. Less common toxicities include myocarditis, encephalitis, uveitis, inflammatory arthritis, and kidney injury. The various toxicities associated with immunotherapy may present clinically as early as several weeks after checkpoint inhibitor initiation, or may not manifest for several years.

Management of suspected irAEs typically includes ruling out infectious causes of symptoms, delaying further immunotherapy, and initiating treatment with corticosteroids. Endocrinopathies related to checkpoint inhibitors are often irreversible and require indefinite hormone replacement therapy. There are no prospective trials guiding management of irAEs. Future studies should aim to identify subpopulations of patients most at risk for developing severe irAEs, and rigorous trials should evaluate optimal management of these toxicities. Clinicians from many specialties must be aware of the spectrum of immune-related toxicities associated with checkpoint inhibitors, as these medications are being used to treat an increasing number of cancer patients, and immunotherapy holds immense promise in the field of medical oncology.

\section{REFERENCES}

1. Postow MA, Sidlow R, Hellmann MD. Immune-related adverse events associated with immune checkpoint blockade. N Engl J Med 2018;378:158-168.

2. Puzanov I, Diab A, Abdallah K, et al. Managing toxicities associated with immune checkpoint inhibitors: consensus recommendations from the Society for Immunotherapy of Cancer (SITC) Toxicity Management Working Group. J Imm Cancer 2017:5:95.

3. Weber JS, Yang JC, Atkins MB, Disis ML. Toxicities of immunotherapy for the practitioner. J Clin Oncol 2015;33:2092-2099

4. Hodi FS, O'Day SJ, McDermott DF, et al. Improved survival with ipilimumab in patients with metastatic melanoma. N Engl J Med 2010;363:711-723

5. Robert C, Schachter J, Long GV, et al. Pembrolizumab versus ipilimumab in advanced melanoma. N Engl J Med 2015:372:2521-2532.

6. Wolchok JD, Neyns B, Linette G, et al. I pilimumab monotherapy in patients with pretreated advanced melanoma: a randomised, double-blind, multicentre, phase 2, dose-ranging study. Lancet Oncol 2010;11:155-164.

7. Pagès C, Gornet JM, Monsel G, et al. I pilimumab-induced acute severe colitis treated by infliximab. Melanoma Res 2013:23:227-230.

8. Kim KW, Ramaiya NH, Krajewski KM, et al. Ipilimumab associated hepatitis: imaging and clinicopathologic findings. Investig New Drugs. 2013;31:1071-1077. 
9. Larkin J, Chiaron-Sileni V, Gonzale R, et al. Combined nivolumab and ipilimumab or monotherapy in untreated melanoma. N Engl J Med 2015:373:23-34

10. Topalian SL, Hodi FS, Brahmr JR, et al. Safety, activity, and immune correlates of anti-PD-1 anti-body in cancer. N Engl J Med 2012;366:2443-2454

11. Naidoo J, Wang X, Woo KM, et al. Pneumonitis in Patients Treated With Anti-Programmed Death-1/Programmed Death Ligand 1 Therapy. J Clin Oncol 2017:35:709-716.

12. Khunger M, Rakshit S, Pasupuleti V, et al. Incidence of Pneumonitis With Use of Programmed Death 1 and Programmed Death-Ligand 1 Inhibitors in Non-Small Cell Lung Cancer: A Systematic Review and Meta-Analysis of Trials. Chest 2017:152:271-281.

13. Weber JS, Hodi FS, Wolchok JD, et al. Safety profile of nivolumab monotherapy: a pooled analysis with advanced melanoma. J Clin Oncol 2017:35:785-792
14. Ryder M, Callahan M, Postow MA, et al. Endocrine-related adverse events following ipilimumab in patients with advanced melanoma: a comprehensive retrospective review from a single institution. Endocr Relat Cancer 2014:21:371-381.

15. Faje AT, Sullivan R, Lawrence D, et al. Ipilimumab-induced hypophysitis: a detailed longitudinal analysis in a large cohort of patients with metastatic melanoma. J Clin Endocrinol Metab 2014:99:4078-4085.

16. Barroso-Sousa R, Barry WT, Garrido-Castro AC, et al. Incidence of Endocrine Dysfunction Following the Use of Different Immune Checkpoint Inhibitor Regimens: A Systematic Review and Meta-analysis. JAMA Oncol 2018:4(2):173-182

17. Hughes J, Vudattu N, Sznol M, et al. Precipitation of autoimmune diabetes with anti-PD-1 immunotherapy. Diabetes Care 2015; 38(4):e55-e57.

18. Nishijima T, Shachar S, Nyrop N, Muss H. Safety and Tolerability of PD-1/ PD-L1 Inhibitors Compared with Chemotherapy in Patients with Advanced Cancer: A Meta-Analysis. Oncologist 2017:4:470-479.

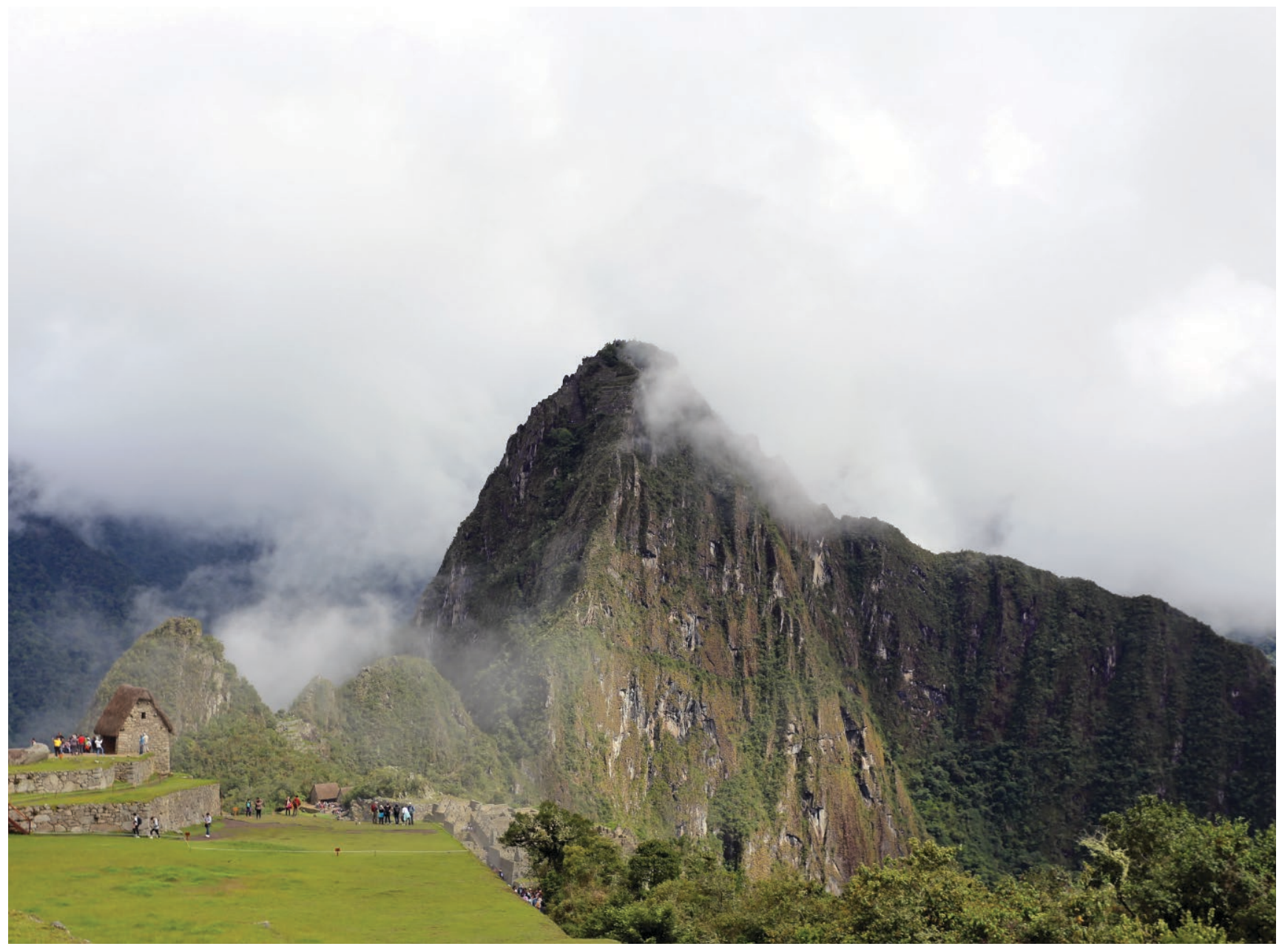

